\title{
Estimación de la postura corporal en un sistema embebido Raspberry Pi empleando técnicas de visión artificial
}

\author{
Fecha de recepción: 23-11-2020 • Fecha de aceptación:05-01-2021 • Fecha de publicación: 10-02-2021
}

\author{
Carlos Luis Vargas Guevara ${ }^{1}$ \\ Universidad Técnica de Ambato, Ecuador \\ cvargas0028@uta.edu.ec \\ https://orcid.org/0000-0003-2050-2342 \\ David Omar Guevara Aulestia ${ }^{2}$ \\ Universidad Técnica de Ambato, Ecuador \\ dguevara@uta.edu.ec \\ https://orcid.org/0000-0002-0410-4398 \\ Marco A Ciaccia ${ }^{3}$ \\ Universidad Técnica del Norte, Ecuador \\ mciaccia@utn.edu.ec \\ https://orcid.org/0000-0001-8293-8040 \\ Thalia San Antonio ${ }^{4}$ \\ Universidad Técnica de Ambato, Ecuador \\ t.sanantonio@uta.edu.ec \\ https://orcid.org/0000-0002-8724-0650
}

\section{RESUMEN}

Los sistemas que se emplean para la captura de movimiento o conocidos como análisis de marcha, generalmente emplean dispositivos dedicados que en el mercado se comercializan a elevados precios. En los últimos años, las empresas enfocadas en la fabricación e implementación de laboratorios de marcha han centrado sus esfuerzos en crear dispositivos de medición y emisión de reportes que sean portables y ocupen un mínimo de espacio; sin embargo, las innovaciones tecnológicas plan- 
teadas por empresas dedicadas al campo de la biomedicina resultan ser cerradas y con limitaciones de compatibilidad. Es decir, que en casos en los que se requiera la expansión del laboratorio, resulta necesario la adquisición de equipos de la misma marca, lo que implica costos elevados. Ante esta problemática, se plantea el uso de sistemas embebidos Raspberry $\mathrm{Pi}$, que, a través de técnicas y librerías de visión artificial, establecen un análisis de marcha con precisión y mayores prestaciones al ser un sistema de código abierto. Asimismo, a través de técnicas de visión artificial se pueda capturar y procesar una imagen binarizada. El empleo de librearías OpenCV para ubicar marcadores y delimitar una región de interés consolidan un sistema compacto y portable para capturar el movimiento corporal en tiempo real, y en espacios no restringidos.

\section{PALABRAS CLAVE: binarización, imágenes, numpy, OpenCV, Raspberry Pi.}

\section{ABSTRACT}

Systems that are used for motion capture or known as gait analysis generally employ dedicated devices that are sold on the market at high prices. In recent years, companies focused on manufacturing and implementing gait laboratories have focused their efforts on creating measurement and reporting devices that are portable and take up a minimum of space; however, technological innovations proposed by companies dedicated to the field of biomedicine turn out to be closed and with compatibility limitations. That is to say, in cases where the expansion of the laboratory is required, it is necessary to acquire equipment of the same brand, which implies high costs. In view of this problem, the use of Raspberry Pi embedded systems is proposed, which, through artificial vision techniques and libraries, establish a running analysis with precision and higher performance as it is an open source system. Likewise, through artificial vision techniques, a binary image can be captured and processed. The use of OpenCV libraries to locate markers and delimit a region of interest consolidate a compact and portable system to capture body movement in real time, and in unrestricted spaces. 


\section{Introducción}

Una de las actividades más importantes para el ser humano es la agricultura, misma que en ocasiones demanda de gran esfuerzo físico para quienes la realizan. Esto se debe a que las personas adoptan posturas forzadas, ejecutan una rutina repetitiva y sobre todo la manipulación de cargas muy pesadas que provocan diversos trastornos musculares y óseos. El levantamiento de cargas muy pesadas se considera como una de las causas principales en los trastornos musculoesqueléticos como son dolores de espalda crónicos y lumbares, que permiten llevar un control de movimientos articulares durante actividades diarias normales (Faisal et al, 2019). A nivel global, los trastornos musculoesqueléticos (TME) han alcanzado un gran impacto en la salud de los trabajadores, influyendo en los procesos de productividad. La Organización Mundial de Salud (OMS) los ha definido como alteraciones de los músculos, tendones, esqueleto óseo, cartílagos, ligamentos y nervios (Comisión Nacional de Seguridad y Salud en el Trabajo, 2008), (Arenas-Ortiz \& Cantú-Gómez, 2013).

En la Tabla 1 se presentan las principales teorías que describen los trastornos mencionados.

Tabla 1.

Teorías de los Trastornos Musculoesqueléticos

\begin{tabular}{|c|c|}
\hline \multicolumn{2}{|l|}{ Teorías de los TME's } \\
\hline Interacción multivariable & $\begin{array}{l}\text { - Componentes individuales } \\
\text { - Propiedades mecánicas: } \\
\text { Dotación genética del individuo } \\
\text { Características morfológicas } \\
\text { Composición psicosocial } \\
\text { Riesgos biomecánicos }\end{array}$ \\
\hline Diferencial de fatiga & $\begin{array}{l}\text { - Actividades ocupacionales desequilibradas } \\
\text { y asimétricas }\end{array}$ \\
\hline Carga acumulativa & - Umbral de carga y repetición \\
\hline Sobreesfuerzo & - $\quad$ Excede el límite de tolerancia \\
\hline
\end{tabular}

Fuente: elaboración propia

El padecimiento de los trastornos musculoesqueléticos es muy diverso, no obstante, existen diferentes teorías que tratan de describir su origen, síntomas y tendencias. Es así, que, la teoría más acertada es suponer que las lesiones musculoesqueléticas son de naturaleza biomecánica. En España, los padecimientos musculoesqueléticos presentan mayor frecuencia en personas dedicadas a realizar actividades repetitivas y de gran esfuerzo físico, seguidos por personas que realizan servicios sociales y de salud (Coloma, 2019).

La Organización Mundial de la Salud (OMS), ha realizado importantes estudios que son asociados al análisis de postura corporal donde resalta que los TME's son la causa principal de la 
discapacidad, destacando al dolor lumbar como una de las fuentes principales de discapacidad a escala mundial. Asimismo, la OMS señala que estos no aparecen solo en adultos mayores, sino a cualquier edad. Lo que influye directamente en la movilidad y destreza de las personas y en algunos casos provocan jubilaciones anticipadas, afectando la participación en la vida social. En el esquema de la Figura 1, se presentan las afectaciones principales de los TME's (Sandoval, 2017).

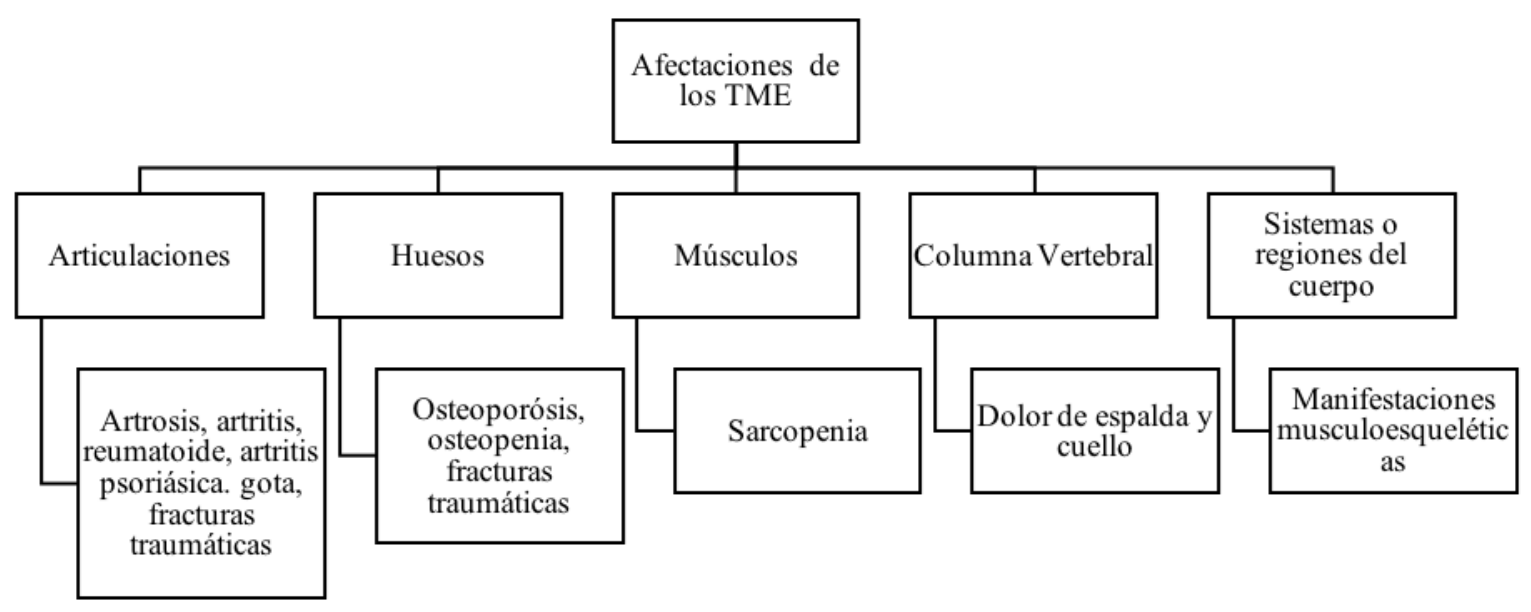

Figura 1. Principales afectaciones de los Trastornos musculoesqueléticos.

Fuente: elaboración propia con información de Sandoval (2017).

Por otra parte, la biomecánica y ciencias del deporte tienen un elevado interés en el análisis de movimiento. Ante ello, se pretende evaluar la postura corporal de las personas y plantear mecanismos de corrección que minimicen los riesgos ocasionados por los TME's (Organización Mundial de la Salud, 2019), (Luttmann, Jager, Griefahn, \& Caffer, 2004).

El continuo desarrollo de herramientas informáticas y sistemas de comunicación humano máquina han consolidado importantes sistemas de captura y análisis de posturas corporales. En forma general, existen diversos sistemas de captura de movimiento o análisis de marcha; pese a esto, la mayor parte de este tipo de soluciones tecnológicas representan a grandes empresas con impacto internacional y costos elevados. En este sentido, la presente propuesta de investigación plantea una solución más económica e innovadora utilizando una tarjeta electrónica Raspberry $\mathrm{Pi}$, una cámara web y técnicas de visión artificial para realizar un análisis de la postura corporal, para ello el trabajo ha sido estructurado en tres etapas: modelo propuesto que describe la implementación y ejecución del sistema de análisis de movimiento, la segunda etapa comprende un análisis y discusión de las implicaciones técnicas y económicas, finalmente se finaliza con las conclusiones alcanzadas y los enfoques que pueden contribuir a futuras investigaciones.

\section{Metodología}

El estudio presenta una propuesta de investigación que plantea el uso de una tarjeta electrónica Raspberry Pi como el núcleo del sistema de captura de movimiento. En el diagrama de la Figura 
2 se describe que el procesamiento de imágenes es una forma de procesamiento de señales que toma como entradas una fotografía, imagen o video; más una unidad de procesamiento de información que para esta investigación es una Raspberry Pi 3B.

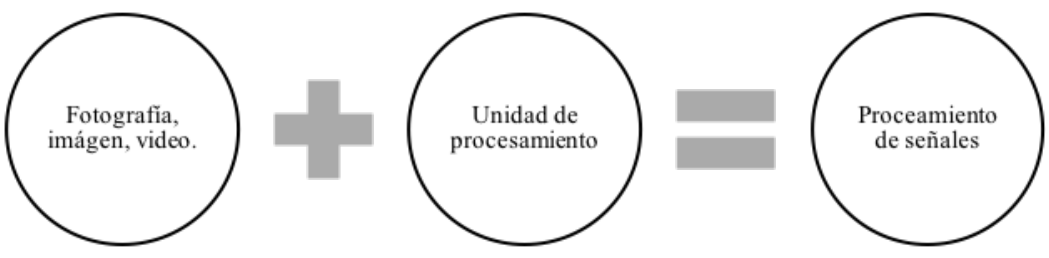

Figura 2. Esquema del procesamiento de señales propuesto.

Fuente: elaboración propia

Es importante que la unidad de procesamiento detecte y manipule un conjunto de características o parámetros relacionados con la imagen en cuestión. El proceso de adquisición de datos se encuentra afectado por sacudidas indeseables y movimientos inestables de la cámara, por lo que es importante emplear algoritmos que mejoren su resolución. Los algoritmos se ejecutan en una tarjeta Raspberry Pi 3B en el entorno de programación de Python, las características de la tarjeta electrónica se presentan en la Tabla 2 basada en investigaciones de Vergara, T. A., \& Dixon, C. S. G. (2005) y de Guerrero Aguirre \& Ramos Giraldo (2014).

Tabla 2.

Características de una Raspberry Pi

\begin{tabular}{|l|l|}
\hline Características de Raspberry Pi \\
\hline Desarrollado por: & Fundación Raspberry Pi \\
\hline Sistema & Broadcom BCM2835 \\
\hline Núcleo & ARM 1176JZF-S (ARM V6K) \\
\hline CPU & $700 \mathrm{MHz}$ \\
\hline GPU & Broadcom Video Core IV 17 pines \\
\hline Potencia & $3,5 \mathrm{~W}$ \\
\hline Memoria RAM & Desde 512 MB (modelo 3B 1Gb) \\
\hline Sistema Operativo & Debian \\
\hline
\end{tabular}

Fuente: elaboración propia

El cuerpo humano es considerado como una estructura mecánica, compuesto por 206 huesos de diferentes formas y tamaños unidos a través de articulaciones. De esta manera los huesos tienen estabilidad y movilidad. Es así como se han desarrollado novedosos sistemas para capturar señales en estos puntos de flexibilidad del cuerpo humano, pero el uso de sensores se ve limitados 
por una serie de factores que se asocian al rendimiento, costo, calibración, etc. (Shilpashree et al, 2015). Así mismo, una desventaja de los sensores inerciales es que son muy sensibles a cambios en los campos magnéticos, en contraste, los sistemas ópticos pueden capturar un elevado número de marcadores a una frecuencia aproximada de 2000 cuadros por segundo (D.A. Bravo, 2016).

En los estudios de biomecánica se consideran como principales articulaciones de locomoción humana la cadera, rodilla, tobillos y metatarso del pie.

De manera general, el modelo que se propone es un sistema de captura del movimiento que a través de la librería de visión artificial OpenCV se programe en Python la captura de coordenadas y puntos de referencia para realizar estimaciones de la postura corporal de una persona. En la Figura 3 se puede ver un diagrama de flujo del modelo propuesto.

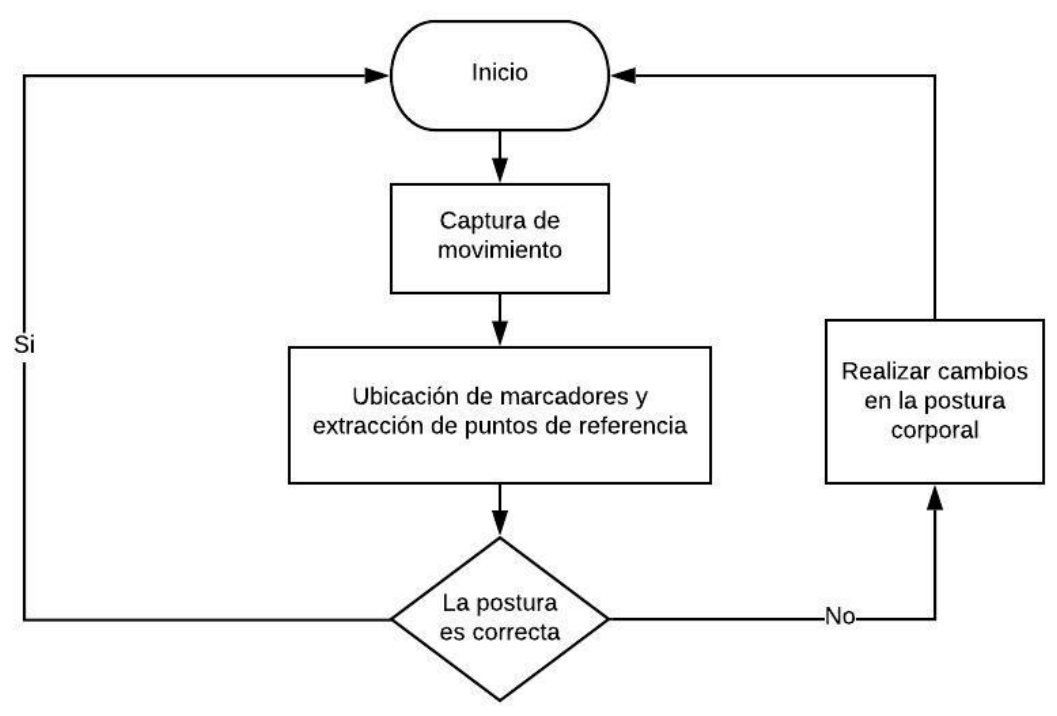

Figura 3. Flujograma propuesto para la captura de movimiento.

Fuente: elaboración propia

En este artículo se ha desarrollado un sistema de detección de movimiento y estimación de la postura corporal. Para ellos se realiza la extracción de puntos de referencia asociados a articulaciones. A través de la programación en Python y empleando la librería de visión OpenCV se pueden extraer los puntos esqueléticos de movimiento, para un rendimiento óptimo es importante considerar el análisis con una unidad de procesamiento gráfico (GPU); sin embargo, se puede realizar con una unidad central de procesamiento (CPU), lo que disminuye la velocidad en su proceso (Raju, 2020), (Pérez, Cavanzo \& Villavisán, 2018).

En el sistema de detección se emplean efectos convexos para lo cual se realizan operaciones sobre contornos, la visión por computadora es muy utilizada sobre todo en áreas de detección y reconstrucción de la estructura esquelética humana. Es así como se emplea una cámara que 
identificará las partes humanas y construirá las articulaciones de la persona que se encuentra en frente de la misma. En este sentido, se ha definido una región de interés de esta manera se limita el procesamiento de los datos para encontrar puntos de interés en dicha área.

La información capturada se procesa a través de pixeles para estimar la posición de las articulaciones, no obstante, los píxeles del contorno pueden causar una gran interferencia, por tal razón se emplea un método de estimación de un núcleo gaussiano ponderado.

\section{Resultados}

Una de las aplicaciones más habituales para el análisis de marcha o biomecánica son los sistemas de captura de movimiento, por ello en la Figura 4, se presenta la clasificación de los mismos (Raju, 2020)

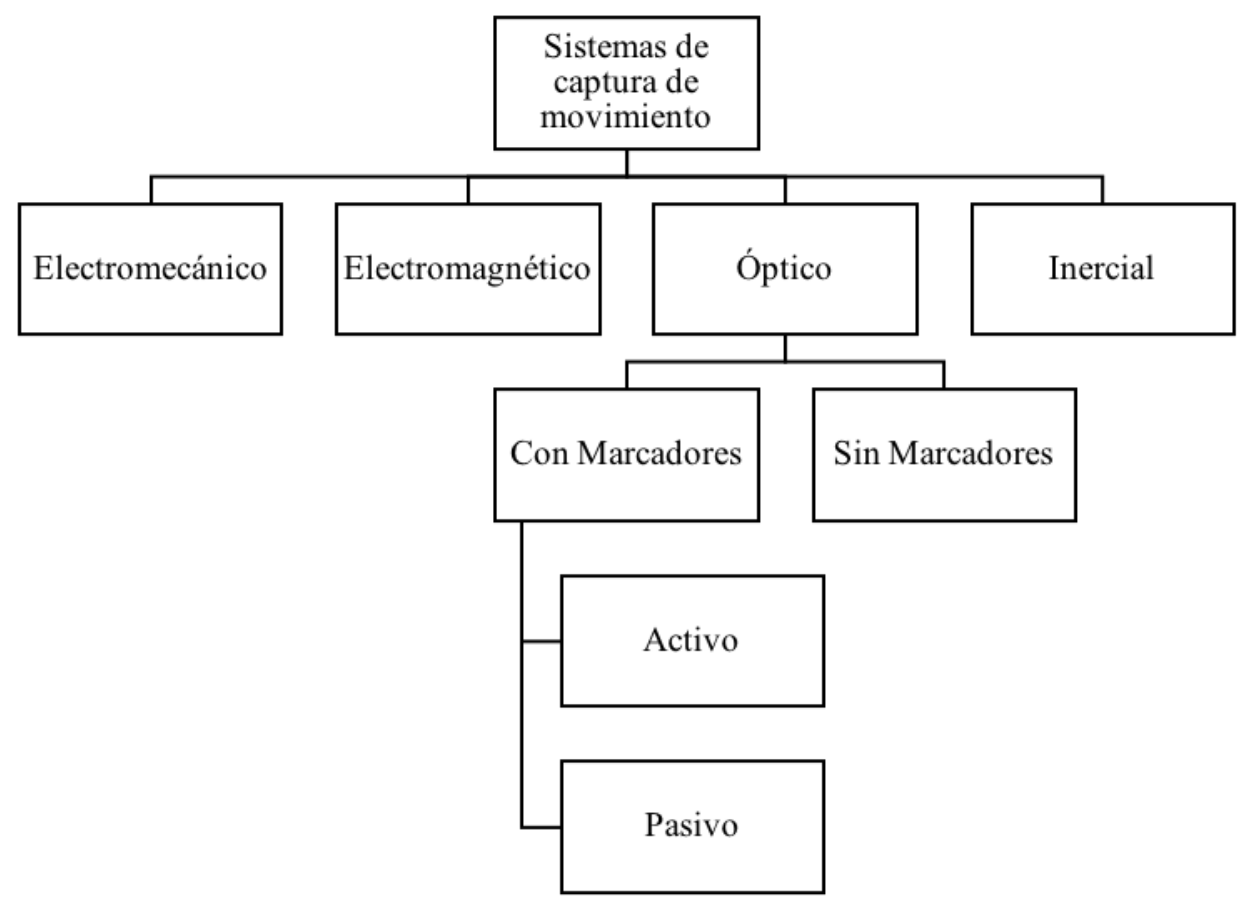

Figura 4. Clasificación de los sistemas de captura de movimiento.

Fuente: elaboración propia

Los sistemas electromecánicos, electromagnético e inerciales incluyen diferentes dispositivos que son invasivos para el cuerpo humano, es así, que, si se adquieren datos, es necesario colocarlos sobre la superficie humana, lo que puede producir incomodidad y limitación del movimiento en forma natural.

En la Tabla 3 se evidencian las principales ventajas y desventajas asociadas al empleo de un sistema óptico de captura sin marcadores. 
Tabla 3.

Ventajas y desventajas de los sistemas de captura de movimiento sin marcadores

\begin{tabular}{|c|c|}
\hline \multicolumn{2}{|c|}{ Sin Marcadores } \\
\hline Ventajas & Desventajas \\
\hline No invasivos & Reconstrucción menos completa \\
\hline Instalación y uso más simple & Menor Precisión \\
\hline Menor coste & \\
\hline
\end{tabular}

Fuente: elaboración propia

Por esta razón, se propone el uso de un sistema óptico que no emplea marcadores, de esa manera se busca capturar el movimiento de manera más habitual y ordinaria, ya que así los movimientos se registran sin que el usuario o paciente en cuestión tenga que llevar equipamiento adicional.

A su vez, se propone el uso de una tarjeta Raspberry Pi para el procesamiento de imágenes que a través de una cámara USB conforman el sistema de adquisición de imágenes, Figura 5.

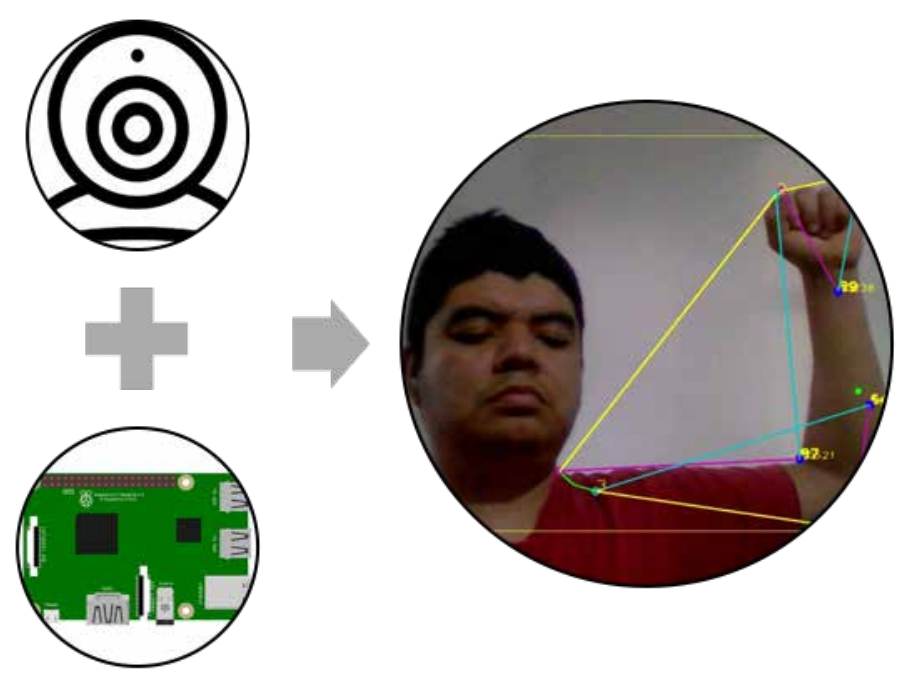

Figura 5. Esquema del sistema de captura de movimiento.

Fuente: elaboración propia

El empleo de una Raspberry Pi es muy funcional, ya que es un sistema embebido de código abierto y que en un futuro se puede integrar a un conjunto de dispositivos o sensores de movimiento, consolidando así un sistema robusto para la captura de movimiento.

La programación del sistema de captura de movimiento se compiló en Python empleando la librería de visión artificial OpenCV, facilitando el procesamiento de imágenes ya sea de forma real, 
a través de streaming, mediante el comando cv2.VideoCapture. Asimismo, se debe ubicar la región de interés que está limitada por un rectángulo de color amarillo, de esa manera el análisis se enfoca en esta área.

También a través de la técnica de umbralización simple, se puede mejorar la resolución de una imagen binaria a través del comando cv2.medianBlur. Con la umbralización se busca segmentar las imágenes separando el área de interés del fondo de la imagen capturada, es así, que en color blanco aparece la imagen binaria, mientras que el fondo permanecerá en color negro. Una recomendación importante es elegir un fondo que contraste bien con las imágenes que se van a capturar, minimizando así los problemas que se pueden presentar al momento de capturar el movimiento corporal.

Una vez que se ha extraído la imagen binaria es importante limitar su contorno, es así, como se procede a calcular las coordenadas $X, Y$ que corresponderán al centro de la imagen en análisis. En la Figura 6 se aprecia la captura de movimiento corporal.

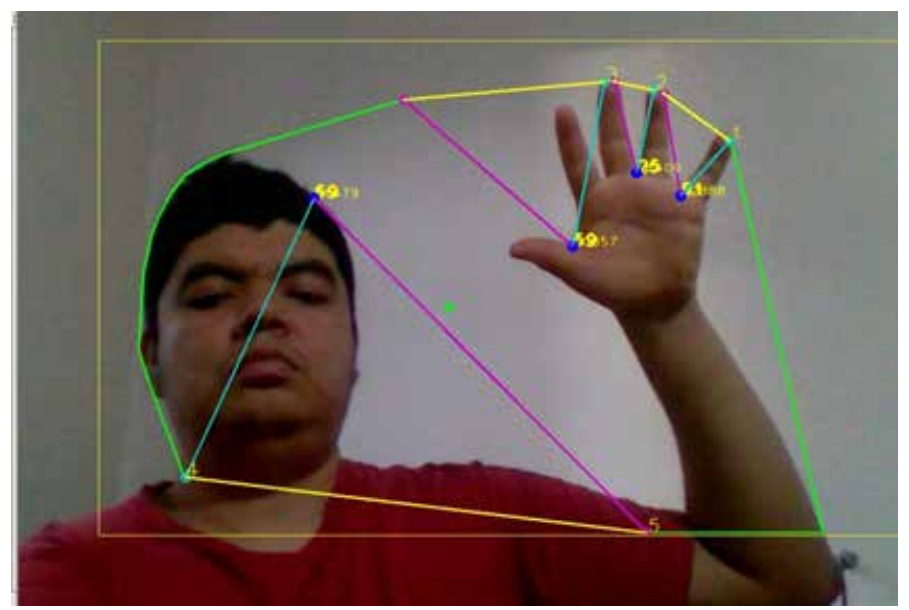

Figura 6. Sistema de captura de movimiento.

Fuente: elaboración propia

De la imagen anterior, se puede apreciar que el área de interés se encuentra limitada por un color amarillo, mientras que en color verde se encuentra dibujado el contorno de la imagen binaria, así también el centro de esta en un punto del mismo color. En color azul se encuentran los puntos de convexidad para estimar las articulaciones y los ángulos de apertura de esta. Para calcular los ángulos se emplea la siguiente ecuación (1).

$$
C=\operatorname{arcCos}\left(\frac{a^{2}+b^{2}+c^{2}}{2 a b}\right)
$$

En la Figura 7 se puede apreciar el código que se utiliza para determinar los ángulos en base a las posiciones convexas formadas por las articulaciones del cuerpo humano. En la librería Numpy se pueden encontrar los lados a, b y c, en primera instancia los ángulos medidos están en radianes, 
por lo que es necesario realizar la conversión a grados para posteriormente expresarlos en pantalla con formato de enteros.

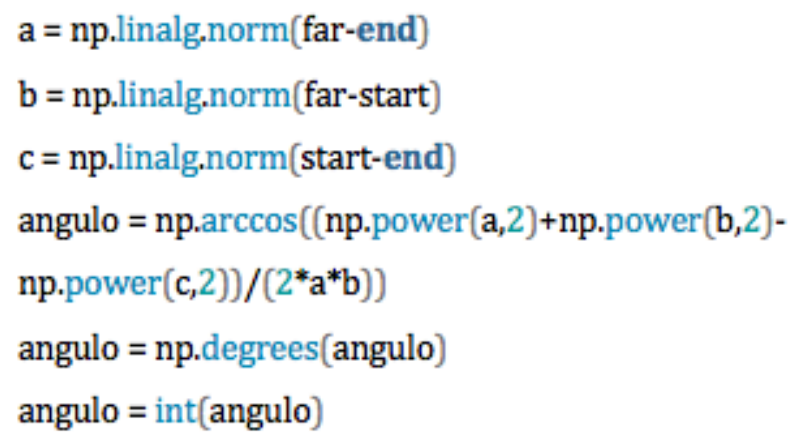

Figura 7. Código para estimar los ángulos en zonas convexas.

Fuente: elaboración propia

El desarrollo del presente trabajo ha permitido capturar los movimientos corporales que posteriormente se pueden exportar a un software dedicado para transformar las señales obtenidas en señales de control. De igual manera, el proyecto permitirá diferentes ampliaciones que proporcionen nuevas formas de comunicación entre emisor y receptor con el fin de obtener un sistema autónomo.

\section{Conclusiones}

En la propuesta realizada se puede utilizar un sistema ligero y económico que permite capturar el movimiento corporal en tiempo real en entornos no restringidos.

A través de un ajuste de distancias, se pudo estimar las posiciones articulares humanas en función de las coordenadas cartesianas en base al proceso de extracción de una imagen binaria.

Un aspecto importante es el sistema de comunicación, ante ello la Raspberry Pi resulta como la mejor opción de comunicación ya que se puede enlazar a través de Bluetooth o WiFi, es decir, una comunicación sin hilos cubriendo así un área de cobertura considerable.

A futuro, se prevé integrar el sistema de captura de movimiento con otros dispositivos del tipo de electromiografía y sensores de fuerza que proporcionen un análisis biomecánico más detallado que pueda ser usado en aplicaciones personales e industriales.

\section{Agradecimientos}

Los autores expresan sus más sinceros agradecimientos al Consorcio Ecuatoriano para el Desarrollo de Internet Avanzado CEDIA, por su especial apoyo en el desarrollo de la presente propuesta, gracias a la financiación del proyecto CEPRA X-III "LLANKAK WARMIKUNATA KAMACHIKKUNA (PROTEGER A LA MUJER TRABAJADORA)" 


\section{Referencias}

Arenas-Ortiz, L., \& Cantú-Gómez, Ó. (2013). Factores de riesgo de trastornos músculo-esqueléticos crónicos laborales. Medicina Interna de México, 370-379.

Coloma Coloma, M. A. (2019). Trastornos musculoesqueléticos de posible origen laboral asociado a posturas forzadas en el personal operativo de limpieza del Ministerio de Agricultura y Ganadería del Ecuador-Quito. Facultad de Ciencias del Trabajo y Comportamiento Humano, 1(1), 1-6.

Comisión Nacional de Seguridad y Salud en el Trabajo. (2008). Enfermedades Profesionales de los Agricultores. Grupo de Trabajo "Sector Agrario", 1(1), 1-36.

D.A. Bravo M, C. R. (2016). Comparación de dos Sistemas de Captura de Movimiento por medio de las Trayectorias Articulares de Marcha. Revista Mexicana de Ingeniería Biomédica, 37(2), 149-160.

Faisal, A. I., Majumder, S., Mondal, T., Cowan, D., Naseh, S., \& Deen, M. J. (2019). Monitoring methods of human body joints: State-of-the-art and research challenges. Sensors, 19(11), 2629. https://doi.org/10.3390/ s19112629

Guerrero Aguirre, A., \& Ramos Giraldo, P. J. (2014). Sistema embebido de bajo costo para visión artificial. Scientia et Technica Año XIX, 163-173.

Luttmann, A., Jager, M., Griefahn, B., \& Caffer, G. (2004). Prevención de Trastornos muscoloesqueleticos en el lugar de trabajo. Berlín: Serie protección de salud de los trabajadores.

Organización Mundial de la Salud. (9 de Agosto de 2019). Trastornos musculoesqueléticos. Recuperado el 22 de Junio de 2020, de https://www.who.int/es/news-room/fact-sheets/detail/musculoskeletal-conditions

Pérez, M., Cavanzo Nisso, G. A., \& Villavisán Buitrago, F. (2018). Sistema embebido de detección de movimiento mediante visión artificial. Visión Eléctróncia, 97-101. https://doi.org/10.14483/22484728.15087

Raju J.B.V.P., Reddy Y.C., G P.R. (2020) Smart Posture Detection and Correction System Using Skeletal Points Extraction. In: Satapathy S.C., Raju K.S., Shyamala K., Krishna D.R., Favorskaya M.N. (eds) Advances in Decision Sciences, Image Processing, Security and Computer Vision. Learning and Analytics in Intelligent Systems, vol 3. Springer, Cham. https://doi.org/10.1007/978-3-030-24322-7_23

Sandoval, S. (2017). Trastornos musculoesqueléticos de origen laboral en el cuello y las extremidades superiores de los fisioterapeutas en Cataluña. Cataluña: Universitat de Lleida.

Shilpashree, K. S., Lokesha, H., \& Shivkumar, H. (2015). Implementation of image processing on Raspberry Pi. International Journal of Advanced Research in Computer and Communication Engineering, 4(5), 199-202. https://doi.org/10.17148/IJARCCE.2015.4545 
Vergara, T. A., \& Dixon, C. S. G. (2005). Sistema de reconocimiento de posturas del cuerpo humano. Pontificia Universidad Javeriana., 1 (1), 1-6. 